\title{
Skills of using Google Maps smartphones application for Geography teachers
}

\section{Maha Kamal Hefny}

curriculums \& teaching methods of Geography Assisstant professor

Education faculty-Assuit University

$$
\text { ورقة عمل بعنوان }
$$

" مهارات استخدام تطبيق خرائط جوجل للهواتف الذكية لمعلمى الجغرافيا " 


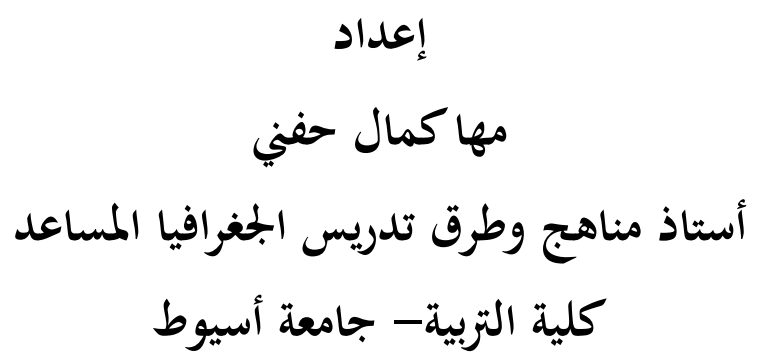

مستخلص الورقة

هدفت هذه الورقة إلى تحديد مهارات استخدام تطبيق خرائط جوجل Google Maps Application للهواتف الذكية بنظام أندروبد اللازمة لمعلمي الجغرافيا ، فمع التطور التكنولوجي كان لابد من توظيف التكنولوجيا وخاصة تكنولوجيا الهواتف الذكية في التعليم وخاصة في مجال التدريس ولا سيما في تدريس الجغرافيا ؛ ومع ظهور العديد من تطبيقات الجغرافيا الرقمية بالهواتف الذكية كتطبيق خرائط جوجل ؛ ساعد ذلك في تسهيل التعرف على الكثير من دول العالم والعواصم والتضاريس والمعالم ، لهذا يتحتم على معلمي الجغرافيا الآن أن يُواصلوا تطوير وتجديد مهاراتهم ومعرفتهم لكيّ يُجاروا الابتكارات المستمرة والتطورات الجديدة في العالَم الرقميّ ؛ الأمر الذي يتطلب تحديد مهارات استخدام تطبيق خرائط جوجل للهواتف الذكية اللازمة لمعلمي الجغرافيا . الكلمات المفتاحية : تطبيق خرائط جوجل- الهواتف الذكية - معلمي الجغرافيا - مهارات استخدام تطبيق خرائط جوجل للهواتف الذكية .

\title{
Skills of using Google Maps smartphones application for Geography teachers Assisstant professor / Maha Kamal Hefny ${ }^{(1)}$
}

\begin{abstract}
This paper aimed to determine the necessary skills of using Google Maps smartphones application with the Android system for geography teachers, as with the technological development it was necessary to employ technology, especially smart phone technology, in education, especially in the field of teaching, especially in the teaching of geography; With the emergence of many digital
\end{abstract}

(2) curriculums \& teaching methods of Geography Assisstant professor - Education faculty-Assuit University. 
geography applications on smartphones, such as the Google Maps application ; This helped facilitate the identification of many countries in the world, capitals, terrain and landmarks, so it is imperative that geography teachers now continue to develop and renew their skills and knowledge in order to keep pace with the continuous innovations and new developments in the digital world; This requires identifying the skills of using the Google Maps smartphones application for geography teachers .

Keywords : Google Maps application - Smart phones - Geography teachers - Skills of using Google Maps smartphones application .

لقد أصبحت التكنولوجيا تُنَخََم لتوصيل المعارف وتتمية مهارات وقيم بطرقٍ جديدةٍ ومبتكَرة ، وبما أن العصر الحالي هو عصر التطور التكنولوجي فلابد من توظيف التكنولوجيا في تعليم وتعلم الجغرافيا ؛ فمن الضروري تطوير تعليم الجغرافيا ، وإدخال التقنيات الحديثة ضمن مناهج الجغرافيا ، والخطط الدراسية ؛ لتلبية حاجات المتعمين , وحاجات سوق العمل . ومن هذه التقنيات الحديثة استخدام التطبيقات التكنولوجية في التدريس وخاصة تطبيقات الهاتف المحمول ؛ حيث تُعد مادة الجغرافيا أحد المواد التي تحتاج إلي دراسة عميقة ، وقد يكون من الصعب على المعلين توصيل كل المعلومات عن الظواهر الجغرافية للطلاب بطريقة سهلة ؛ حيث لا يمثل فتح الأطلس المطبوع نفس متعة اكتثاف خريطة ثلاثية الأبعاد يتم عرضها على شاشة جهاز ، ولكن بفضل التكنولوجيا وتطور الهواتف الذكية أصبح الأمر أسهل بكثير على كل من المعلمين والطلاب على حد

وأحد هذه التطبيقات هو تطبيق خرائط جوجل Google Maps Application . وهو " أداة عامة لرسم الخرائط ، والاستفادة من طبيعتها المرئية للغاية لإنثاء أداة مساعدة متخصصة لفهم التوجيه ومفاهيم نظم المعلومات الجغرافية الأخرى مثل الترميز الجغرافي وتحديد الموقع الجغرافي " • (Cheong et al , 2012) لهذا برزت الحاجة لتعلم مهارات تدريسية جديدة ، حيث يتحتم على معلمي الجغرافيا الآن أن يُواصلوا تطوير وتجديد مهاراتهم ومعرفتهم لكيّ يُجاروا الابتكارات المستمرة والتطورات الجديدة في العالَم الرقميّ ؛ الأمر الذي يتطلب تحديد المهارات التكنولوجية اللازمة لاستخدام تطبيق خرائط جوجل للهواتف الذكية لمعلمي الجغرافيا .

Technology of smart phones : تكنولوجيا الهواتف الذكية

مع التطور التكنولوجي والمعلوماتي ؛ أصبحت مهارات تكنولوجيا المعلومات والإتصالات مدخلا لعالم اليوم ، والأشخاص 
الذين لا يمتلكون تلك المهارات من المرجح عدم قدرتهم علي التواصل مع هذا العالم (Dekelver et al,2015,283) • وأحد مجالات تكنولوجيا الدعلومات والإتصالات هو الهاتف الذكي وهو : " هاتف محمول عادة ما يكون له واجهة تعمل باللمس (Oxford Living " والوصول إلي الإنترنت ونظام تثغيل قادر علي تثغيل التطبيقات أو التطبيقات التي يتم تتزيلها

. Dictionaries , 2018)

وتتنمي تقنيات الهواتف الذكية إلى تكنولوجيا أجهزة الحاسوب المحمولة متعددة الأغراض ، وهي تتميز بتكنولوجيا متعددة تمكنها من تشغيل وظائف الوسائط المتعددة بما في ذلك الموسيقى والفيديو والكاميرات والألعاب بجانب وظائف الهاتف الأساسية مثل المكالمات الصوتية والرسائل النصية ، وتثتمل الهواتف الذكية عادةً على أجهزة استشعار مختلفة يمكن الاستفادة منها بالإضافة إلى دعم بروتوكولات الاتصالات مثل البلوتوث والواي فاي والتتقل عبر الأقمار الصناعية ، كما أن من مزايا الهواتف الذكية استخدام العديد من التطبيقات المختلفة والمفيدة . www.wikiwand.com) ومع ظهور العديد من التطبيقات بالهواتف الذكية خاصة التطبيقات الجغرافية ؛ ساعد ذلك في تسهيل التعرف على الكثير من دول العالم والعواصم والتضاريس والمعالم ؛ ومن هذه التطبيقات تطبيق خرائط جوجل . | تطبيق خرائط جوجل للهواتف الذكية : Google Maps smartphones application تعد خرائط جوجل في الأساس خدمة مجانية مقدمة من موقع البحث جوجل على الثبكة العالمية ، وتوفر الخدمة عند بدايتها عرضُ لخرائط لشوارع أربع دول هي : الولايات المتحدة وكندا والمملكة المتحدة وأيرلندا محَ إمكانية تحديد مكان عمل ما في تلك الدول ، هذا بِالإضافة إلى استطاعة الثخص مشاهدة صور عالية الوضوح بواسطة الأقمار الصناعيّة لمئات من المُدن في العالم ، أمَّا الآن فلقد توسعت شبكة الطرق والثوارع لتشمل مُعظم مُدن العالم وليس الأربعة التي بدأ بها المشروع فقط (https://ar.wikipedia.org/wiki) مشهد الثارع أو حركة المرور ، كما تسمح بجولات افتراضية لمواقع معينة علي سطح الأرض . (https://support.google.com/maps/answer/144349?hl=ro\&ref topic=3092425) وتتميز خرائط جوجل بالعديد من الخدمات التي تجمع البيانات الرقمية لتضفي مزيدًا من الواقعية على استخدام الخرائط مثل خدمة جوجل إيرث وخدمة تصميم الخرائط ، فهذه من أهم خدمات جوجل التي تمنح المستهلك أفضلية التعامل مع الخرائط من حيث (www.quora.com) . إضافة الطرق وغير ذلك ومع التطور في تكنولوجيا الهواتف الذكية تحولت تلك الخرائط إلي تطبيقات يمكن أن يستخدمه الفرد علي هاتفه أينما ذهب وفي أي 
وقت · حيث تثهد متاجر التطبيقات الآن ظهور عدد كبير من التطبيقات التعليمية ومنها التطبيقات الجغرافية والتي تجعل من تعلم الجغرافيا أمرا ممتعًا ، وأحد هذه التطبيقات هو تطبيق خرائط جوجل ـ وتتوفر خرائط جوجل كتطبيق للهواتف المحولة لأنظمة تشغيل أندرويد وآي أو إس (Vanlerberghe,2008) وذكرت صحيفة الغارديان أنَ جوجل كانت تُعدّ تطبيق "خرائط جوجل" الخاص بها لنظام آي أو أس ، وعندما أصدرته في 12 ديسمبر 2012 ، تمَّ تنزيل التطبيق أكثر من عشرة ملايين مرَّة خِلال يومين فقط ، وفي نهاية عام 2015 أعلنت خرائط جوجل عن وظائفها الجديدة غير المتصلة بالإنترنت ؛ حيثُ يُمكن تنزيل خرائط بمساحة 120 ألف كيلومتر مربع كدد أقصى لاستخدامها لاحقاً دون الاتصال بالإنترنت ، ويتطلّب ذلك قدرًا كبيرًا من مساحة التخزين ، وفي ديسمبر 2017 أضافت جوجل وضعًا جديدًا للدراجات النارية مصمم للمستخدمين في الهند ، يسمح بمزيد من إمكانية الوصول في ظروف حركة المرور ، كما أنه في عام 2019 قدم إصدار أندرويد ميزة جديدة تُسمى العرضُ المباشر يتيح عرضُ الاتِّجَاهَاتِ مباشرةً على الطريق بفضل الواقع المعزز ، وفازت خرائط جوجل بجائزة "Webby 2020" لأفضل واجهة مستخدم في فئة التطبيقات والجوال والصوت .

\section{(https://ar.wikipedia.org/wiki)}

وعليه ؛ يسمح تطبيق خرائط جوجل لنظام اندرويد للمستخدمين بمشاهدة سطح الأرض من خلال صور وخرائط الأقمار الصناعية ، ورؤية المدن من أعلى ومن خلال عرض الشوارع ، والبحث عن العناوين وتصفح المواقع ، ويحتوي علي العديد من المباني والهياكل الثهيرة من جميع أنحاء العالم بمناظر ثلاثية الأبعاد مفصلة يمكن الوصول إليها ، وتتيح العروض التاريخية للمستخدمين رؤية التغييرات في الأماكن على الأرض بمرور الوقت ، وتقديم فهم لأجزاء مختلفة من العالم للطلاب ، و Google Google store هو تطبيق مجاني يمكن لأي فرد تنزيله علي هاتفه الدحمول من مخزن التطبيقات maps. الفرق بين تطبيق خرائط جوجل وبعض التقنيات الجغرافية الأخري :

1- نظام تحديد المواقع العالمي GPS : يعد نظام الـ GPS واحدا من أبرز المميزات الموجودة فى الهواتق الذكية الحديثة ؛ حيث يقوم بمساعدة المستخدمين على تحديد موقعهم الجغرافى ؛ مع القدرة على مشاركة هذا الموقع ، ويتم كل هذا بنفس الطريقة على جميع الهواتف الذكية بغض النظر عن نظام التشغيل ، وتعمل عادة هذه التقنية لتحديد موقع المستخدم غالبا بدقة تصل إلى 5 أمتار ، وكلمة GPS اختصار لـGlobal Positioning System أى نظام تحديد المواقع العالمى ، وهى تقنية مملوكة فى الأساس للحكومة الأمريكية ، وهى متاحة بشكل مجانى للجميع ، وهى عبارة عن نظام ملاحة لاسلكى يستخدم موجات الراديو بين الأقمار الصناعية وجهاز الاستقبال داخل هاتف المستخدم لتوفير معلومات عن الموقع والوقت لأى نظام 
تثغيل.(حواس،2018،

20 جوجل إيرث Google Eearth : هو برنامج خرائطي وجغرافي معلوماتي كان يطلق عليه في الأصل Earth Viewer 3D ؛ أنشأته شركة كي هول Keyhole وهي شركة امتلكتها جوجل سنة 2004 ، يرسم البرنامج خريطة للأرض عن طريق تركيب الصور التي تم الحصول عليها من صور الأقمار الصناعية ، والتصوير الجوي ونظم المعلومات الجغرافية الثلاثية الأبعاد الخاصة (Gearthblog.com). بالكرة الأرضية

\section{مميزات استخدام تطبيق خرائط جوجل لنظام آندرويد :}

حدد Vanlerberghe ( 2008 ) مميزات تطبيقات جوجل علي الهواتف الذكية والتي منها تطبيق خرائط جوجل كالتالي : 1- سهل الاستخدام : يمكن تسجيل الدخول إلى حساب Google الخاص بك والوصول الفوري إلى جميع تطبيقات Google المفضلة لديك . 2- متزامن بالكامل • يتم دفع الأحداث الجديدة في الوقت الفعلي إلى هاتفك وأي تغييرات تجريها أثناء التتقل متاحة على الفور على الويب . 3- 3 - 3صمة للعمل معًا. كما أن هناك خمسة مميزات رئيسة في خرائط جوجل ( البوابة العببية للأخبار التقنية ، 2020 ) : 1- التحقق من قوائم المطاعم ومراجعات العملاء :

تُعد خرائط جوجل بمثابة تطبيق لاستعراض مراجعات العملاء لمكانٍ ما ومعرفة مدى جودته، بحيث يمكنك الحصول على فكرة جيدة عن أنواع الخيارات المتوفرة في وجهتك ، وتعد هذه الميزة مفيدة بشكل خاص إذا كانت وجهتك عبارة عن مطعم أو فندق ، حيث تساعدك معاينة خيارات القائمة والأسعار بالإضافة إلى المراجعات الفردية للمستخدمين على اتخاذ قرار بالذهاب أو عدمه . 2-2 عمليات الحجز والطلب : بمجرد الحصول على نظرة سريعة على جميع الخيارات التي يقدها للك المطعم الذي تريد الذهاب إليه ، يمكنك حجز طاولة مباشرة من تطبيق (خرائط جوجل) Google Maps بدون الخروج منه ، وإذا كنت تفضل تتاول الطعام في المنزل ، يمكنك أيضًا الطلب من داخل التطبيق . 3- استخدام مساعد جوجل على هواتف آيفون : 
يأتي نظام IOS مع المساعد الصوتي ، ولكن يمكنك من داخل إعدادات خرائط جوجل تفعيل مساعد جوجل للاعتماد عليه أثناء التتقل .

\section{4- معرفة زمن الوصول والانتظار :}

يمكن استخدام خرائط جوجل لمعرفة المدة الزمنية التي يمكنك أن تستغرقها للذهاب والانتظار في مكتب الطبيب ، أو متجر

البقالة ، أو لإنجاز بعض المعاملات الحكومية المبة

\section{5- البحث عن ساعات العمل وأرقام الهواتف :}

إذا احتجت إلى معرفة موعد فتح أحد المتاجر أو المكاتب أو إغلاقه ، أو كنت بحاجة إلى الاتصال بالمكان وليس لديك الرقم

، أو احتجت إلى الرمز البريدي للشركة ، انتقل مباشرة إلى (خرائط جوجل) Google Maps للحصول على هذه الإجابات . |

إعتاد الكثير من الناس على استخدام الخرائط لمعرفة الكثير من المعلومات المفيدة بخصوص الثوارع والطرق والبلاد التي يريدون الوصول إليها ، ومما يسّر ذلك في العصور الحديثة هو سهولة طريقة استخدام الخرائط في الأيفون وفي الأندرويد وغيرهما

من الوسائل الحديثة ، ويمكن توضيح المزيد عن أهمية استخدام تطبيقات الخرائط فيما يأتي (www.quora.com) : - - تمثل تطبيقات الخرائط نافذة حقيقية على بيانات العالم الحقيقي لكن على نطاق أصغر بكثير فهي بمثابة خريطة تكنولوجية تساعد على السفر من مكان إلى آخر.

- - تساعد تطبيقات الخرائط في تتظيم الوقت وتضييق المسافات وإنجاز العمل دون هدر زمن بالغ في الوصول إلى الدكان المقصود.

- - تثكل تطبيقات الخرائط أهمية بالغة في الرحلات والاستكثافات العلمية التي تتضمن بيئتها منحدرات أو انهيارات أرضية. - من أسباب أهمية تطبيقات الخرائط هو إمكانية حساب الوقت اللازم للوصول.

- - تُضفي تطبيقات الخرائط مزيدًا من البهجة عندما يحدد المستخدم المسار الذي يريد المرور من خلاله، فهي توفر له إمكانية أن يتجاوز طريق صحراوي على سبيل المثال ليمر بطريق آخر يمر ببحيرات وسواحل بحرية . وتعد خرائط جوجل حالة تطورية لأدوات الجيل الثاني للإنترنت ، والتي تمثل منصة تعلم تفاعلية ، تتسم قدراتها بالتوافر الدائم والحداثة والمجانية والتقاعلية والإتاحة عبر شبكة الإنترنت وتطبيقات الهواتف النقالة والأجهزة اللوحية ، وتزداد يوما بعد يوم فاعليتها 
في تطوير التدريس التفاعلي لمناهج دراسية مختلفة من أبرزها مناهج الجغرافيا ، إذ أثبتت بعض الدراسات السابقة علي قدرة خرائط جوجل بصفة عامة وخرائط جوجل ماب في تتمية العديد من الأهداف التربوية ، ومن هذه الدراسات : 1 - دراسة Cheong et al (2012): هدفت الدراسة إلي تحديد أثر استخدام خرائط جوجل مع طلاب الدراسات العليا في تحديد الطرق وأفضلها ، وتم تضمينها في طريقة التدريس التي ركزت على التصور كاستراتيجية معرفية ، وتم تدريب الطلاب ، وتم قياس نتائج التعلم وحصل جميع الطلاب على درجات في النطاق ( 7 :10 من 10 ) .

2- دراسة كاظم وعيد ( 2014 ) ؛ وهدفت الدراسة إلي معرفة أثر استعمال الخرائط الأكترونية ( جوجل إيرث وجوجل ماب ) والصور الفضائية عبر الثبكة العالمية في تحصيل واحتفاظ طلاب الصف الثاني المتوسط في مادة الجغرافي بجامعة بابل / كلية التربية للعلوم الانسانية ، زاستغرقت الدراسة (8) أسابيع لتطبيق الدراسة ، وأسفرت النتائج عن تحسين تحصيل واحتفاظ طلاب الصف الثاني المتوسط في مادة الجغرافي نتسجة استخدام الخرائط الأكترونية والصور الفضائية عبر الثبكة العالمية في تدريس داظة · الجغرافي 3- دراسة Galbin (2015) : والتي هدفت إلي تحديد آراء طلاب المرحلة الثانوية حول فوائد استخدام تطبيق خرائط جوجل وتطبيق جوجل إيرث على أجهزة الكمبيوتر اللوحية وأجهزة الكمبيوتر المحمولة في تدريس دروس الجغرافيا ؛ حيث أجري البحث خلال ندوات نادي الجغرافيا التي نظمت في ثانوية "Radu Petrescu" في العام الدراسي 2014/2013 ، وشارك في البحث (13) طالبًا في مختلف درجات الصالة الرياضية والمدارس الثانوية ، طُلب منهم الرد على استبيان بعد إجراء الأنشطة ، وكثفت النتائج علي أن الطلاب استمتعوا باستخدام هذه التطبيقات مع أجهزة الكمبيوتر المحمولة والأجهز اللوحية وأن عملية التعلم خلا دروس الجغرافيا أصبحت أكثر إثارة للاهتمام .

4- دراسة البلوي والحويطي (2017) : وهدفت الدراسة إلي معرفة فاعلية استخدام خرائط جوجل إيرث (Google Earth) للطالبات بالمرحلة الابتدائية في تتمية مهارة تحديد المواقع واتجاهات الطالبات نحو موضوعات الجغرافيا في مقرر التربية الاجتماعية والوطنية بمدينة تبوك ، وتكونت عينة البحث الحالي من (68) طالبة من طالبات الصف الساس الابتدائي من المدرسة (الابتدائية السابعة والعشرون) التي اختيرت بطريقة عشوائية ، وتم توزيع أفراد العينة إلى مجموعتين، إحداهما المجموعة التجريبية (34) طالبة ، تم تدريهن باستخدام خرائط جوجل (Google) الإكترونية ، والمجموعة الثانية المجموعة الضابطة (34) طالبة ، تم تدريهن بالطريقة الاعتيادية ، وأسفرت النتائج عن فاعلية استخدام خرائط جوجل (Google) الإكترونية في تتمية مهارة تحديد المواقع 
الجغرافية واتجاه الطالبات نحو موضوعات الجغرافيا لمقرر التربية الاجتماعية والوطنية لصالح المجموعة التجريبية . 5- دراسة Saleem (2018) : والتي هدفت إلي تحديد تأثير طريقة الرحلة باستخدام خرائط جوجل علي مستويات الاسترجاع أو الذاكرة لدى طلاب البيولوجيا بالمدارس الثانوية في مدرسة ثانوية حضرية في مدينة باترسون ، نيو جيرسي ، تم استخدام اختبار لاحق مكون من ثلاثين سؤالًا على فترات أسبوعين وأربعة أسابيع بعد التدريس لمحتوى التعلم ، وأسفرت النتائج علي ارتفاع مستويات الاسترجاع المعلوماتي لدي الطلاب في البيولوجيا وبقاء أثر التعلم .

6- دراسة الزغيي وقطب ( 2018 ) : هدفت الدراسة إلى تحديد جدوى استخدام التقنيات الجغرافية والتي منها خرائط جوجل في تتمية مهارات قراءة واستخدام الخرائط لاى طلاب الصف الأول المتوسط في المدينة المنورة في مقرر الدراسات الاجتماعية والتربية الوطنية الفصل الأول ، وحدة " الخرائط والتقنيات الحديثة " ، وخلصت الدراسة إلى مجموعة من النتائج أبرزها : توجد فروق ذات دلالة إحصائية عند مستوى (05, ) بين متوسطي درجات طلاب المجموعة التجريبية وطلاب المجموعة الضابطة في التطبيق البعدي لصالح طلاب المجموعة التجريبية في مهارات استخدام مقياس الرسم لقياس الأبعاد والمساحات على الخريطة ، ومهارات استخدام مفتاح الخريطة ، ومهارات تحديد المواقع الجغرافية ، ومهارات استخدام برنامج جوجل إيرث / جوجل ماب .

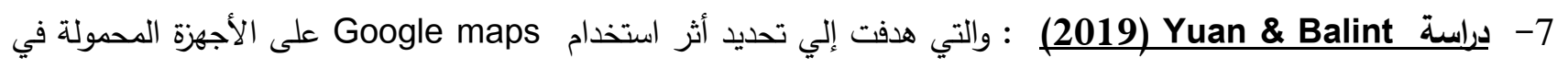
تتمية المهارات اللازمة للتتقل في المجتمعات لدي ثلاثة أفراد من ذوي الإعاقات الذهنية والتتموية (IDD) لما بعد المرحلة الثانوية ، وأظهرت النتائج أن اثثين من المشاركين تعلما جميع الخطوات لاستخدام خرائط جوجل ، كما نجحوا في الوصول إلى الوجهات بثكل مستقل باتباع الطرق التي خططوا لها. ومن نتائج الدراسات السابقة يتضح قدرة خرائط جوجل علي زيادة الدافعية للتعلم وبقاء أثر التعلم وتتمية مهارات قراءة وفهم واستخدام الخرائط الرقمية وتحديد المواقع وتتمية التفكير المكاني إضافة إلي تتمية إتجاهات إيجابية نحو تعلم المادة وربطها بحل مشكلات الحياة ، من هنا تأتي أهمية التركيز علي خرائط جوجل واستخدامها بالصفوف التعليمية ومن خلال أنشطة مادة الجغرافيا ، الأمر الذي يتطلب إلمام معلم الجغرافيا بالمهارات التكنولوجية اللازمة لاستخدام تطبيق خرائط جوجل للهواتف الذكية .

\section{| كيفية استخدام تطبيق خرائط جوجل للهواتف الذكية :}

مع ظهور الاتجاه العالمي باستخدام تكنولوجيا المعلومات في التدريس والتعلم ؛ ومع زيادة اهتمام المعلمين والمتعلمين باستخدام الأجهزة المحمولة كالهواتف الذكية لصفر حجمها وزيادة سعة تخزينها للمعلومات عن طريق الوسائط المتعددة والوصول 
إلي الإنترنت والثبكات الاجتماعية وكذلك التطبيقات ؛ ساعد استخدام التطبيقات علي الهواتف المحمولة الذكية علي تحسين العملية التعليمية وزيادة التواصل متعدد التخصصات بين المعلومات التي يتم الحصول عليها أثناء التعلم . لهذا كان من الضروري محو الأمية الرقمية (Hall et al , 2014) • وإعداد وتدريب المعلمين علي استخدام هذه التطبيقات ؛ حيث إن تدريب وإعداد المعلمين على الاستخدام الهادف لكل ما تقدمه التكنولوجيا المعاصرة لتطوير المناهج الدراسية يتطلب يقظة مستمرة وتجريبًا وابتكارًا ومراجعة وتحديثًا .

كما دعت بعض الدراسات إلي ضرورة محو الأمية الجغرافية لدي الأفراد كدراسة (Lisle , 2006) ، ودراسة , Thomas) (Chou et al , 2012) ( 2011 ، ودراسة (الأمر الذي يتطلب استخدام التطبيقات الجغرافية لتحقيق أهداف التعليم الجغرافي . وأحد هذه التطبيقات هو تطبيق خرائط جوجل ؛ حيث توفر خرائط جوجل للتعليم موارد لمساعدة المعلمين والطلاب علي استكثاف أدوات رسم الخرائط وابتكارها والتعاون معها (http://maps.google.com/help/maps/education ) لهذا أوصت بعض الدراسات كدراسة (Nosir,2015) ، ودراسة ( البلوي والحويطي ، 2017) ، ودراسة الزغيبي وقطب ( 2018 ) ، ودراسة (Williams \& Shekhar,2019) علي ضرورة تدريب وإعداد المعلم علي استخدام الخرائط التفاعلية كخرائط جوجل من أجل التدريس الفعال للجغرافيا . وتعد خرائط جوجل من أكثر خدمات شركة جوجل شعبية ، بالإضافة إلى الميزات والدقة العالية التي توفرها ، فإن استخدامها يمكن أن يتجاوز المساعدة في توفير الخرائط للتتل فقط ، حيث إنها تُخفي بين طياتها الكثير من الميزات والأدوات القوية ، والبيانات المختلفة التي يمكنك اكتثافها والاستفادة منها ، وفيما يلي خطوات استخدام تطبيق خرائط جوجل في الهواتف الذكية علي نظام أندرويد Android ، وتم اختيار نظام تشغيل أندرويد لأنه الأسهل في الاستخدام والأكثر انتشارا كنظام تشغيل للهواتق الذكية: أ- تحميل وتنزيل تطبيق خرائط جوجل من Google play .
جوجل
خرائط
تطبيق
استخدام
ب- أساسيات

: (https://support.google.com/maps/answer/6139433?hl=ar\&ref_topic=3092425) • حفظ عنوان المنزل والعمل عن طريق : - فتح تطبيق "خرائط - Google" - النقر على الأماكن المحفوظة ، حفظ مكان ضمن "قوائمك" ، النقر على الأماكن المصنّفة. 
• حفظ الأماكن المفضّلة : يمكن حفظ الأماكن المفضلة على الخريطة وذلك للعثور عليها سريعًا في وقت لاحق من أي جهاز كمبيوتر أو هاتف أو جهاز لوحي. - لحفظ الأماكن وعرضها على "خرائط Google"، التأكد من تسجيّل الدخول إلى حساب Google. - يمكن تصنيف عنوان لم يتم تحديده في "خرائط Google"، مثل منزل أحد الأقارب ، للعثور عليه بسهولة .

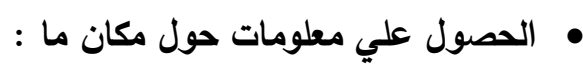
- تحديد مكانًا على الخريطة ؛ للحصول على الاتجاهات إليه ـ أو الاطلاع على معلومات ، مثل ساعات العمل ، والقوائم وشاهد

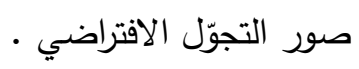
- فتح تطبيق "خرائط Google - خرائط - البحث عن مكان أو النقر عليه في الخريطة.

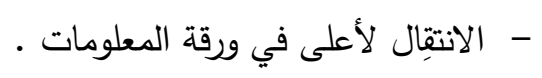
• • بدء التنقل : - فتح تطبيق "خرائط Google". - - الحصول على الاتجاهات إلى موقع جغرافي.

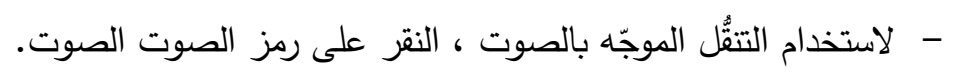
- للخروج من التتقُل، في أسفل يمين الثاشة، النقر على رمز الإغلاق X ج-عند فتح تطبيق "خرائط Google ، ستجد (5) علامات تبويب في أسفل "الثشاشة الرئيسة (https://support.google.com/maps/answer/9625865?hl=ar\&ref_topic=3092425\&visit_id=637 :462964412191174-391898128\&rd=1) 1-استكثاف : لاختيار الأماكن المراد الذهاب إليها . 2- انتقال Go: ما يمكن توقّعه أثناء التتقّلات اليومية المتكررة . 3- أماكن محفوظة : حفظ مكان لإنشاء قوائم وتذكُر أماكن . 
4- المساهمة : لمشاركة تجارب ، وإضافة معلومات وتعليقات ، وحلّ مشاكل . 5- المستجدّات : لتلقّي إشعارات بالمعلومات ذات الصلة .

- تتوفّر ميزات مثل "مشاركة الموقع الجغرافي" و"المخطط الزمني" والخرائط المتوفّرة بلا إنترنت في رمز صورة الملفت الثخصي أو الحرف الأوّل من الاسم دائرة الحساب في أعلى يسار الثاشة.

• إدارة إعدادات الخصوصية : النقر على صورة الملف الثخصي أو الحرف الأوّل من الاسم دائرة الحساب للوصول إلى إعدادات الخصوصية وإدارتها كالتالي : - المخطط الزمني: للاطّلاع على معلومات "سجلّ المواقع الجغرافية" وإدارتها . - مشاركة الموقع الجغرافي : لاختيار من يمكنه الاطّلاع على موقعك الجغرافي ومن يمكنك الاطّلاع على موقعه الجغرافي على "خرائط "Google" - بياناتك في "خرائط Google": لإدارة عناصر التحكّم في الخصوصية . د- تثغيل تطبيق خرائط جوجل في جميع الهواتف الذكية بنظام تشغيل أندرويد في أي مكان وبدون إنترنت كالتالي ( مدونة : معلوم ، د.ت ) - - تحميل وتشغيل برنامج خرائط جوجل مجانا : تمن متجر جوجل play ثم قم بتحديث التطبيق إلى آخر اصدار . - Offline Area فتح التطبيق والضغط على - Select Your Own Area ثم الضغط علي - مownload تحديد المنطقة الجغرافية المراد تشغيلها مجانا ثم الضغط - - بعد تحميل الحزمة المطلوبة وبعد الانتهاء يمكن تثغيل التطبيق مجانا وبلا انترنت . هـ - وقد استعرضت ( البوابة العربية للأخبار التقنية ، 2020 ) بعضا من خطوات استخدام تطبيق خرائط جوجل في الهواتق

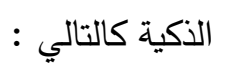
• تفعيل مساعد جوجل للاعتماد عليه أثناء التنقل باتباع الخطوات التالية : - - فتح تطبيق (خرائط جوجل) - - النقر على أيقونة القائمة (ثلاثة خطوط أفقية على يسار شريط البحث) . 


$$
\text { - - - انلقر على خيار (الإعدادات) - }
$$

- التمرير للأسفل ، ثم اختر (الوصول إلى المساعد باستخدام “OK Google” ) ، حرك مفتاح التبديل. • معرفة زمن الذهاب والانتظار في مكان ما كالتالي : - - في تطبيق (خرائط جوجل) انلقر على الموقع المراد زيارته . - الانتقال للأسفل حتى رؤية رسمًا بيانيًا يُسمى (الأوقات الثائعة) Popular times -

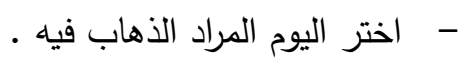
- - الضغط على العمود الأحمر للاطلاع على بيانات حركة المرور الحالية . - - تحت العنوان ، قم بتخطيط زيارتك ، وستجد معلومات عامة مثل : أوقات الانتظار القصوى ، وطول مدة الزيارة المتوسطة . • البحث عن موعد بدء أي عمل وأرقام الهواتف ومواقع الويب كالتالي : - - ساعات العمل : تُعرض ساعات عمل الشركات أسفل العنوان مباشرة ، النقر فوق السهم في الأسفل بجانب وقت إغلاق اليوم

$$
\text { لمشاهدة ساعات العمل في الأيام الأخرى من الأسبوع. }
$$

- أرقام الهواتف : بعد الجزء المخصص لساعات العمل في معظم القوائم يوجد رقم الهاتف ، للاتصال به دون مغادرة تطبيق الخرائط ، النقر فوق الرقم ، ثم النقر فوق النافذة المنبثقة التي تعرض المطالبة بالاتصال متبوعة بالرقم . - - موقع الويب : إذا كان لاى الثركة موقع ويب ، فيوجد أسفل رقم الهاتف ، النقر على الرابط لفتح موقع الويب في علامة تبويب على المتصفح · و - ومن خطوات استخدام "خرائط Google" على الهاتف المحمول البحث والاستكثاف والحصول على اتجاهات للوصول إلى أي العالم

$$
\text { في }
$$

مكان

:=(https://support.google.com/maps/answer/144349?hl=ar\&ref_topic=3092425\#zippy) • التعرُّف على آلية عمل الخريطة : يمكن استخدام "خرائط Google" على الهاتف المحول بطرق متعدّدة. • العثور على نتائج البحث : يمكن البحث عن مكان أو نوع أماكن أو العثور على عمليات البحث الأخيرة أو الحصول على اتجاهات ـ وتوجد نتائج البحث على شكل دبابيس صغيرة حمراء ونقاط حمراء ؛ حيث تعرض الدبابيس الصغيرة أهم النتائج ، وللاطّلاع على مزيد من المعلومات أو الحصول على اتجاهات أو الاطّلاع على نشاط تجاري من الداخل ، النقر على أي نتيجة. 
• استكثاف الخربطة : على الهاتف أو الجهاز اللوحي ، النقر مع الاستمرار على أي مكان على الخريطة للحصول على تفاصيل بشأنه .

• قياس المسافات بين النقاط : يمكن قياس المسافات بين نقطتَين كالتالي : - الخطوة الأولي : إضافة النقطة الأولى :

- - على هاتقك أو جهازك اللوحي الذي يعمل بنظام التشغيل Google Android ، افتح تطبيق خرائط Google خرائط - المس مع الاستمرار أي مكان في الخريطة ـ ستلاحظظهور دبوس أحمر • - في الجزء السفلي ، انقر على اسم المكان • - في صفحة المكان ، مرّر لأسفل واختر قياس المسافة . - الخطوة الثانية : إضافة النقطة أو النقاط التالية :

- حرِّك الخريطة بحيث تكون الدائرة السوداء أو المؤشر المتقاطع فوق النقطة التالية التي تريد إضافتها . - في أسفل اليسار ، انقر على إضافة إضافة . - - أضف كل النقاط التي تريدها. - الخطوة الثالثة : الحصول على المسافة : - في الجزء السفلي ، سترى المسافة الكلّية بالأميال أو بالكيلومترات . - اختياري : لإزالة النقطة الأخيرة التي أضفتها، انقر على تراجع تراجع. لمحو كل النقاط: في أعلى اليسار، انقر على المزيد المزيد ثم محو. - - عندما تتنهي من ذلك، انقر على سهم الرجوع أعلى اليمين. • الإبلاغ عن مشكلة متعلقة بالبيانات : ساعِد في تحسين "خرائط Google" من خلال الإبلاغ عن المشاكل التي تلاحظها في الخريطة ، مثل تفاصيل غير صحيحة عن أنشطة تجارية أو أسماء طرق غير صحيحة أو أخطاء أخرى كالتالي : - إضافة معلومات عن الأماكن أو تعديلها : يمكنك إضافة الأماكن غير المُدرَجة على الخريطة لتكون متاحة للجميع، مثل إضافة المعالم العامة أو المقاهي أو الأنثطة التجارية المحلية الأخرى إلى الخريطة ، كما يمكن الاطّلاع على مزيد من المعلومات عند إضافة مكان غير مدرَج على "خرائط Google" ، فيمكنك إضافة معلومات عن مكان أو نشاط تجاري أو تعديلها ، مثل : الاسم أو العنوان أو ساعات العمل أو حقائق أخري . 
- إضافة عناوين أو تصحيحها : ويمكنك إضافة عناوين بريدية أو تعديلها لتكون متاحة للجميع أو تصحيح مواقع تسليم الطرود أو تعديل مواضع الدبوس على مواقع جغرافية. - إضافة طريق أو تصحيحه : إذا كنا بحاجة إلى إضافة طرق إلى "خرائط Google"، أو كان هناك خطأ في الخريطة، يمكنك إخبارنا بذلك. - إزالة بيانات غير صحيحة أو احتيالية : يمكنك الإبلاغ عن أنشطة تجارية أو أسماء أماكن في حال انتحال الهوية أو عرض محتوى مسيء أو زائف أو غير مرغوب فيه أو غير ملائم. - الاطّلاع على تعديلاتك ومعرفة حالتها : على هاتفك المحمول، يمكنك الاطّلاع على قائمة بالتعديلات والأماكن التي أضفتها إلى "خرائط Google". وتكون تعديلاتك مرئية لك فقط. بعض الأماكن - التي أضفتها وتظهر على أنها موافق عليها • الاطّلاع على المعلومات المتعلّة بحركة المرور والنقل العام وركوب الاراجات والتضاريس ومعلومات حول فيروس كورونا المستجد (كوفيد-19) : (19) - - على جهازك الجوّال، افتح تطبيق "خرائط Google" خرائط Google. في أعلى الصفحة، انقر على رمز الطبقات الطبقات ثم حدّد خيارًا. • مشاهدة العالم بعرض ثلاثي الأبعاد : لمشاهدة عرض Earth الثلاثي الأبعاد على الهاتف، انتقِل إلى اعلي يسار الثاشة وانقر على المربّع. • الاطّلاع على ميزة "التجوّل الافتراضي" : إذا كانت صور "التجوّل الافتراضي" متوفرة، ستجد صورة مصغّرة لميزة "التجوّل الافتراضي" عندما تختار مكانًا على الخريطة ، وباستخدام ميزة التجوّل الافتراضي، يمكنك استكثاف معالم العالم، ومشاهدة عجائب الدنيا الطبيعية، والدخول إلى أماكن مثل المتاحف والساحات والمطاعم والأنشطة التجارية الصغيرة. ويمكنك استخدام ميزة التجوَل الافتراضي في خرائط Google كالتالي : 1- ابحث عن مكان أو أسقط دبوسًا : - - انقر على محدّد موقع المكان. - البحث عن مكان أو إسقاط دبوس - افتح تطبيق خرائط Google - ابحث عن مكان أو أسقط دبوسًا على الخربطة. لإسقاط دبوس، المس الخربطة مطولاً. 
- في الجزء السفلي، انقر على اسم المكان أو العنوان.

- مرّر لأسفل وحدد الصورة التي تسمى "التجوّل الافتراضي أو حدد الصورة المصغرة التي تحتوي على رمز التجوّل الافتراضي . - - عند الانتهاء من ذلك، انتقل إلى أعلى اليمين وانقر على "رجوع •

2- انقر على محدّد موقع المكان :

- افتح تطبيق خرائط Google -

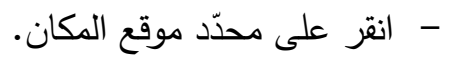
- في الجزء السفلي، انقر على اسم المكان أو العنوان.

- مرّر لأسفل وحدد الصورة التي تسمى "التجوّل الافتراضي أو حدد الصورة المصغرة التي تحتوي على رمز التجوّل الافتراضي . - - عند الانتهاء من ذلك، انتقل إلى أعلى اليمين وانقر على "رجوع • 3-مشاركة صورة تجوّل افتراضي : لمشاركة صورة التجوّل الافتراضي، انقر على الثاشة، ثم انقر على "المزيد" > مشاركة. 4- استكشاف التجوّل الافتراضي :

- لاستكثاف ما حولك في التجوّل الافتراضي، اسحب إصبعك على الثاشة أو انقر على البوصلة. - للتجوّل، مرّر سريعًا جهة اليمين أو اليسار • يمكنك أيضًا التمرير بسرعة لأعلى أو لأسفل على الخط الأزرق. - لمشاهدة عرض أوسع والتغيير إلى الوضع الأفقي، يمكنك تحريك جهازك إلى أحد الجانبين. - للتكبير ، اضغط مع فتح الإصبعين على الثاشة. للتصغير، اضغط مع غلق الإصبعين على الثاشة. • تحريك الخربطة : للعثور على مزيد من التفاصيل على الخريطة، يمكنك التكبير أو التصغير • على جهازك الجوّال، يمكنك استخدام الإيماءات مثل التدوير والإمالة والتكبير أو التصغير للتنقّل بسرعة في تطبيق "خرائط Google" ، ويُكنك تحريك الخريطة بيد واحدة كالتالي : - افتح تطبيق "خرائط Google" خرائط Google" على هاتفك أو جهازك اللوحي الذي يعمل بنظام التشغيل Android ، ثم اسحب الخريطة بإصبعك. • تغيير حجم النص : يُكنكك ضبط حجم العناوين على الخريطة حتى تتمكن من رؤيتها بوضوح أكثر ، ثم افتح تطبيق "الإعدادات" تطبيق "الإعدادات" على هاتفك أو جهازك اللوحي الذي يعمل بنظام التشغيل Android ، وانقر على أدوات تمكين 
الوصول. ومن هنا، يُكناك :تغيير حجم الخط: لجعل الكلمات أكبر ، انقر على حجم الخط ثم اضبط حجم الخط المفضّل - تغيير حجم العرض: لجعل العناصر على شاشتك أكبر ، انقر على حجم العرض ثم اضبط حجم الرمز المفضّل. • سحب الخربطة : يُكنك تكبير حجم الخربطة وتصغيره بيد واحدة ، افتح تطبيق "خرائط Google" خرائط Google" على هاتفك أو جهازك اللوحي الذي يعمل بنظام التثغيل Android ، انقر مرّتين على أي مكان في الخريطة ، ثم: - - اسحب إلى الأسفل لتكبير الخربطة. - - - - اسحب إلى الأعلى لتصغير الخريطة.

• عديد موقعك الجغرافي الحالي : للبحث عن أماكن من حولك والحصول على الاتجاهات بسرعة أكبر، يمكنك تحديد مركز - - مشاهدة موقعك الحالي على الخريطة موقعك الجغرافي الحالي. - افتح تطبيق خرائط Google خرائط Google على هاتفك أو جهازك اللوحي الذي يعمل بنظام التثغيل Android. - سترى نقطة زرقاء توضّح موقعك الحالي. إذا لم تثاهد النقطة الزرقاء، انتقل إلى الأسفل وانقر على موقعك الجغرافي الموقع. - كيفية عثور خرائط Google على موقعك الجغرافي الحالي ، تقدر الخرائط موقعك الحالي من الموارد مثل: - نظام تحديد المواقع العالمي (GPS): يستخدم هذا الخيار الأقمار الصناعية ويحدد موقعك الجغرافي في محيط 20 مترًا. ملاحظة: عندما تتواجد داخل المباني أو تحت الأرض، فإن نظام تحديد المواقع العالمي (GPS) يكون غير دقيق في بعض الأحيان. Google يساعد موقع شبكات WiFi-Fi - البرج الخلوي: يمكن أن يكون اتصالك بالثبكة الخلوية دقيقًا بما يصل إلى بضعة آلاف من الأمتار. - تشغيل وضع الدقة العالية : استخدم وضع الدقة العالية لمساعدة خرائط Google في العثور على موقعك بنقطة زرقاء أكثر دقةً. - افتح تطبيق الإعدادات الإعدادات على هاتفك أو جهازك اللوحي الذي يعمل بنظام التشغيل Android ، وانقر على الموقع ، شغِل الموقع في الجزء العلوي ، انقر على الوضع ثم دقة عالية . 
كتابة تعليقات وإضافة تقييمات للأماكن : يمكنك كتابة تعليقات على "خرائط Google" عن الأماكن التي زرتها. يمكنك أيضًا إضافة معلومات عن مكان تثير فيها مثلاً إلى أجوائه الهادئة أو الحيوية أو الرومانسية. وثُقدَّم هذه التعليقات والآراء بشكلٍ طوعي، كما أننا لا ندفع مبالغ مالية للمراجعين مقابل إضافتها إلى "خرائط Google". - البيانات التي سيراها الآخرون : تكون التعليقات علنية ، لذا يمكن لأي شخص الاطّّلاع على ما تكتبه. ولا يمكنك إضافة تعليق مجهول الهوية ، وفي ما يلي بعض المعلومات الإضافية التي ستظهر للآخرين : الاسم الذي يظهر على صفحة نبذة عني التعليقات الأخرى التي كتبَتها في "خرائط Google" - الصور التي أضفتها إلى "خرائط Google" .

وبذلك تطبيق خرائط جوجل هو : أداة معلومات جغرافية للتعلم القائم علي نظم المعلومات الجغرافية (GIS) لتحليل الظواهر العلمية والمكانية المعقدة في تعليم الجغرافيا ، مما يؤدي إلي تحسين مهارات التفكير الجغرافي للطلاب والمعلمين ، ومهارات تقسير الخرائط وتحليل البيانات الجغرافية الجية

\section{\# مهارات استخدام تطبيق خرائط جوجل في الهواتف الذكية لمعلمي الجغرافيا :}

مما سبق تم التوصل إلي قائمة بالمهارات التكنولوجية لاستخدام تطبيق خرائط جوجل للهواتف الذكية بنظام تشغيل أندرويد لمعلمي الجغرافيا ، وكانت كالتالي (2) : 


\begin{tabular}{|c|c|c|c|}
\hline \multicolumn{4}{|c|}{ المهارات التكنولوجية لاستذام تطبيق خرائط جوجل للهواتف الذكية بنظام تثغيل أندرويد لمعلمي الجغرافيا } \\
\hline خطوات الإستخدام & 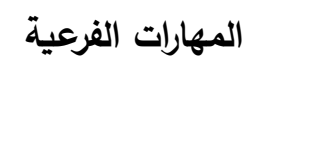 & المهارات & r \\
\hline $\begin{array}{r}\text { google play تثبيت تطبيق خرائط جوجل علي جهاز الموبايل من } \\
\text { store }\end{array}$ & 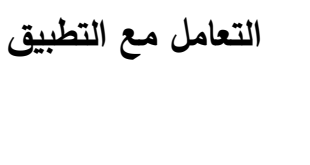 & تكنولية & 1 \\
\hline إزالة تطبيق خرائط جوجل من علي جهاز الموبايل من قائمة التطبيقات & & عامة & 2 \\
\hline كتابة كلمات البحث عن مكان ما في تطبيق خرائط جوجل & & & 3 \\
\hline التمكن من البحث الصوتي عن مكان ما في تطبيق خرائط جوجل & & & 4 \\
\hline محو كلمات البحث السابقة عن مكان ما في تطبيق خرائط جوجل & & & 5 \\
\hline عرض وإخفاء الثريط السفلي بتطبيق خرائط جوجل & & & 6 \\
\hline عرض صور أماكن الزيارة بتطبيق خرائط جوجل & & & 7 \\
\hline عرض صور لأماكن تم استكثافها بتطبيق خرائط جوجل & & & 8 \\
\hline عرض البيانات والنتائج بتطبيق خرائط جوجل & البيانات والمعلومات & & 9 \\
\hline استرجاع المعلومات بتطبيق خرائط جوجل & & & 10 \\
\hline تحديث البيانات يدويا بتطبيق خرائط جوجل & & & 11 \\
\hline طباعة الخرائط من تطبيق خرائط جوجل & & & 12 \\
\hline إرسال بريد إلكتروني يحتوي علي صورة أو خريطة من تطبيق خرائط & & & 13 \\
\hline
\end{tabular}




\begin{tabular}{|c|c|c|}
\hline جوجل & & \\
\hline كتابة تعليق علي خرائط تطبيق خرائط جوجل & & 14 \\
\hline مشاركة الموقع الجغرافي من تطبيق خرائط جوجل & & 15 \\
\hline تعديل البيانات علي خرائط تطبيق خرائط جوجل & & 16 \\
\hline تعديل الملف الثخصي علي تطبيق خرائط جوجل & & 17 \\
\hline تثبيت الدبوس لتحديد مكان الوجهة ( مكان الذهاب إليه ) بتطبيق خراز & التنقل & 22 \\
\hline الإنتقال إلي صفحة اختيار نقطة البداية أثناء التجول من خلال تطبيق & & 23 \\
\hline خرائط جوجل & & \\
\hline تخطيط مسار الحركة بالزمن بوسيلة الإنتقال (عجلة-سيارة-اتوبيسس- & & 24 \\
\hline 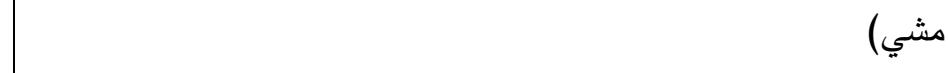 & & \\
\hline التحقق من أفضل المسارات للتتقل بتطبيق خرائط جوجل & & 25 \\
\hline حفظ الأماكن الsفضلة بتطبيق خرائط جوجل & الحفظ الحف & 26 \\
\hline حفظ أماكن مرغوب في زيارتها بتطبيق خرائط جوجل & & 27 \\
\hline حفظ أماكن مميزة بنجمة بتطبيق خرائط جوجل & & 28 \\
\hline حفظ المعلومات التي تم التوصل إليها بتطبيق خرائط جوجل & & 29 \\
\hline حفظ الخرائط في ذاكرة تطبيق حرائط جوجل & & 30 \\
\hline عمل مخطط زمني لأماكن تم زيارتها بتطبيق خرائط جوجل & & 31 \\
\hline إعطاء تفاصيل الطرق الرئيسة والفرعية ومناطق الإهتمام بتطبيق & & 32 \\
\hline خرائط جوجل & & \\
\hline إضافة صور أو خرائط بتطبيق خرائط جوجل & 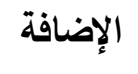 & 33 \\
\hline إضافة مكان علي خرائط تطبيق خرائط جوجل & & 34 \\
\hline تحديد الدستجدات عن المناطق والأماكن التي تتم زيارتها ومتابعتها & 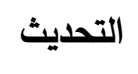 & 35 \\
\hline
\end{tabular}




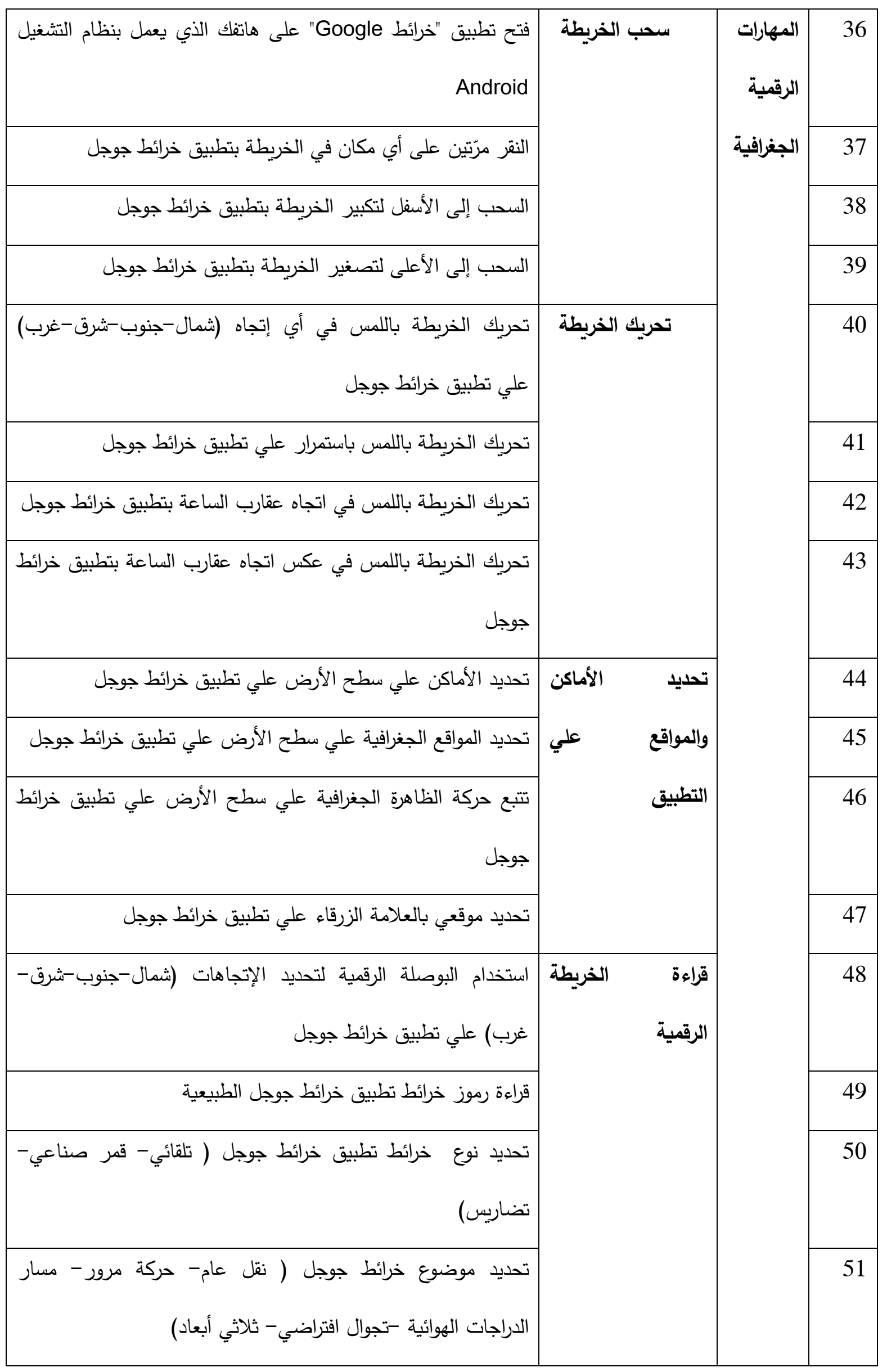




\begin{tabular}{|c|c|c|}
\hline تمثيل البيانات الكمية للخرائط بالألوان علي تطبيق خرائط جوجل & & 52 \\
\hline قراءة رموز خرائط تطبيق خرائط جوجل البشرية & & 53 \\
\hline قراءة مقياس الرسم علي تطبيق خرائط جوجل & & 54 \\
\hline قياس أطوال الأنهار علي تطبيق خرائط جوجل & & 55 \\
\hline تحديد المساحات لأي مكان علي تطبيق خرائط جوجل & & 56 \\
\hline قياس المسافات بين أي مكانين علي تطبيق خرائط جوجل & & 57 \\
\hline تحديد الكثافة المرورية في الوقت الفعلي علي تطبيق خرائط جوجل & & 58 \\
\hline الاستكثاف أماكن قريبة علي تطبيق خرائط جوجل بميزة التجول & علي الخرائط & 59 \\
\hline الاستكثاف أماكن بعيدة علي تطبيق خرائط جوجل بميزة التجول & & 60 \\
\hline استكثاف المكونات الجغرافية للبيئة باستخدام تطبيق خرائط جوجل & & 61 \\
\hline جوجل إنتاج خريطة رقمية ذات تمثيل دقيق لمنطقة ما علي تطبيق خرائط & إنتاج & 62 \\
\hline تفسير الظاهرات الجغرافية علي تطبيق خرائط جوجل & تفسير خرائط التطبيق & 63 \\
\hline استتتاج معلومات جغرافية من تطبيق خرائط جوجل & & 64 \\
\hline المقارنة بين الظاهرات الجغرافية علي تطبيق خرائط جوجل & تحليل خرائط التطبيق & 65 \\
\hline المقارنة بين خرائط تطبيق خرائط جوجل بالأمس واليوم المتغيرة لنفس & & 66 \\
\hline تحليل الظاهرات الجغرافية ثلاثية الأبعاد علي تطبيق خرائط جوجل & & 67 \\
\hline Google فتح تطبيق خرائط & إدخال الإحداثيات & 68 \\
\hline كتابة الإحداثيات في مربّع البحث بالجزء العلوي & ل للعثور على مكان ما & 69 \\
\hline ظهور دبوس عند إحداثياتك & & 70 \\
\hline
\end{tabular}




\begin{tabular}{|c|c|c|}
\hline فتح تطبيق خرائط Google خرائط Google. & \multirow{4}{*}{ إحداثيات أحد الأماكن } & 71 \\
\hline لمس مع الاستمرار منطقة على الخريطة لم يتم تصنيفها & & 72 \\
\hline ظهور دبوس أحمر & & 73 \\
\hline ظهور الإحداثيات في مربّع البحث بالجزء العلوي. & & 74 \\
\hline
\end{tabular}

\section{توصيات الورقة :}

1- تدريب معلمي الجغرافيا علي مهارات استخدام تطبيق خرائط جوجل للهواتف الذكية . 2- إعداد الطلاب المعلمين شعبة جغرافيا علي استخدام تطبيق خرائط جوجل للهواتف الذكية .

3- لفت نظر المسئولين بتخطيط برنامج إعداد معلم الجغرافيا بإضافة مهارات استخدام تطبيق خرائط جوجل Google maps • للهواتف الذكية لـعايير ومحتوي البرنامج application

4- رصد الاحتياجات التدريبية لمعلمي الجغرافيا فيما يخص تكنولوجيا الهواتف الذكية . 5- تخصيص جزء من درجة التقويم في التربية العملية لمستوي إجادة الطلاب المعلمين شعبة جغرافيا لكيفية استخدام تطبيق خرائط جوجل للهواتف الذكية في التدريس • 6- يجب توفير معمل للجغرافيا بكلية التربية ، يمكن للطلاب من خلاله دراسة التطبيقات الرقمية الجغرافية وممارسة مهاراتها سواء فرديا أو جماعيا بشكل إيجابي • 


\section{قائمة المراجع}

أولا : المراجع العربية :

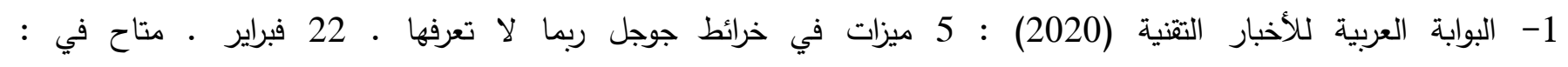
https://aitnews.com/2020/02/22/5-\%D9\%85\%D9\%8A\%D8\%B2\%D8\%A7\%D8\%AA$\% D 9 \% 81 \% D 9 \% 8 A-\% D 8 \% A E \% D 8 \% B 1 \% D 8 \% A 7 \% D 8 \% A 6 \% D 8 \% B 7-$ \%D8\%AC\%D9\%88\%D8\%AC\%D9\%84-\%D8\%B1\%D8\%A8\%D9\%85\%D8\%A7-\%D9\%84\%D8\%A7|\%D8\%AA\%D8\%B9\%D8\%B1\%D9\%81\%D9\%87\%D8\%A7. 2- البلوي ، نوف بنت موسي والحويطي ، رحاب عبد الله (2017) : فاعلية استخدام خرائط جوجل (Google Maps) الإكترونية

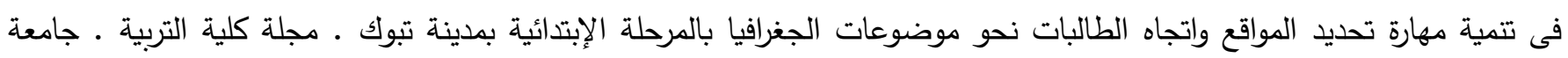

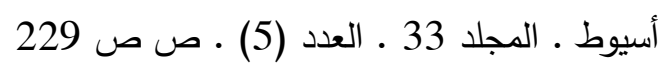
3- الزغيبي ، صالح عبد الله عبد الحميد وقطب ، إيمان محمد مبروك (2018) ـ فاعلية استخدام التقنيات الجغرافية في تتمية المهارات الخاصة بقراءة واستخدام الخرائط ، لاى طلاب الصف الأول المتوسط بالمدينة المنورة ـ مجلة الراسخون الدولية . ع (4) ـ مجلد 2 .

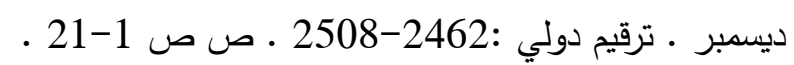

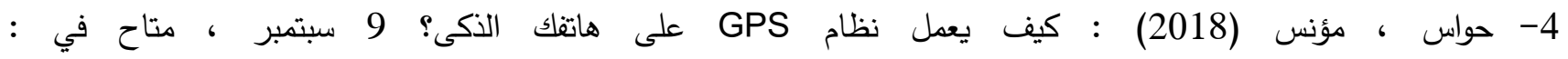
https://www.youm7.com/story/2018/9/9/\%D9\%83\%D9\%8A\%D9\%81\%D9\%8A\%D8\%B9\%D9\%85\%D9\%84-\%D9\%86\%D8\%B8\%D8\%A7\%D9\%85-GPS\%D8\%B9\%D9\%84\%D9\%89-\%D9\%87\%D8\%A7\%D8\%AA\%D9\%81\%D9\%83$\% D 8 \% A 7 \%$ D9\%84\%D8\%B0\%D9\%83\%D9\%89/3942296.

5-كاظم ، شيماء حمزة وعبيد ، محمد فرحان ( 2014 ) ـ أثر استعمال الخرائط الإلكترونية والصور الفضائية عبر الشبكة العالمية

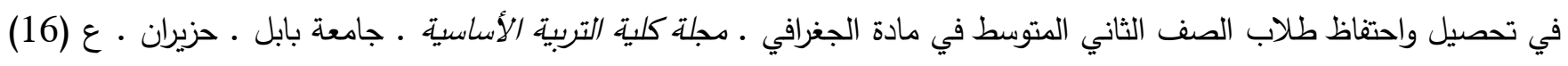

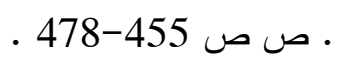
ثانيا : المراجع الأجنبية : 6- Cheong,France ; Cheong,Christopher \& Jie,Ferry (2012) · Re-Purposing Google Maps Visualisation for Teaching Logistics Systems . Journal of Information Technology Education : Innovations in Practice, V(11),p.p 159-177. 
7- Dekelver,J.;Kultsova,M.;Shabalina,O.;Borblik,J.;Pidporigora,A.\&Romanenko,R.(2015).Design of mobile applications for people with intellectual disabilities. In Proceedings of the Creativity in Intelligent Technologies and Data Science Conference (CIT\&DS).Volgograd.Russia.15-17 Sept.pp823-836.

8- Eriksson,Gillian; Weber,Christine \& Kirsch,Lauri (2012) . A Comprehensive Plan for Differentiating the Training of Teachers of the Gifted Online at the State, District and University Levels in Florida , USA .Gifted Education International. Jan.V(28).N 1.p.p41-57.

9- Galbin,Florin (2015) .The Use of Laptop Computers,Tablets and Google Earth/Google Maps Applications during Geography Club Seminars.Romanian Review of Geographical Education.Ej1216205.V(4).N(1).Feb.p.p29-44.

10-Nosair,Mohamed Ragab (2015) • توظيف الخرائط الإكترونية في التدريس التفاعلي لمناهج الجغرافيا بالمدارس QScience Proceedings . Conference on Education . April. Available at : http://dx.dol.org/qproc.2015.coe.39

11- Oxford Living Dictionaries Definition of smartphone (2018).In English Oxford Living Dictionaries : Oxford University Press $\quad$ Availabel at : https://en.oxforddictionaries.com/definition/smartphone.

12-Saleem, Hafiz M (2018) : Memorizing Informational Text Using Journey Method with Google Maps . ProQuest LLC , Ed.D. Dissertation . New Jersey City University . ER/C Number: ED596401. 13-Williams,Peter \& Shekhar,Sidharth (2019) .People with Learning Disabilities and Smartphones : Testing the Usability of a Touch-Screen Interface . Education Sciences .V(9).p263.

14-Yuan,Chengan \& Balint-Langel,Kinga (2019) : Effects of Constant Time Delay on Route Planning Using Google Maps for Young Adults with intellectual and Developmental Disabilities .Education and Training in Autism and Developmental Disabilities.V(54),N 3,Sep.p.p 215-224.

15- Vanlerberghe, Marc (2008) . Google on Andriod. Google mobile blog.September. available at: http://googlemobile.blogspot.se/2008/09/google-on-android.htm/ .

\section{ثالثا : المواقع الإكترونية :}

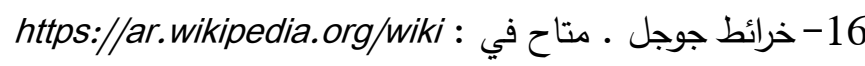

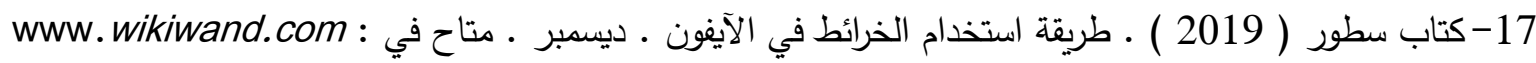

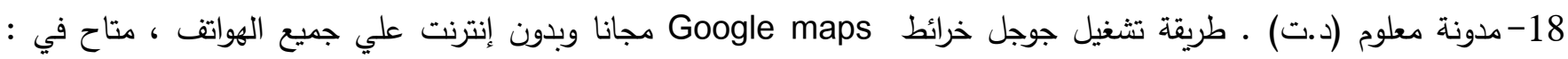

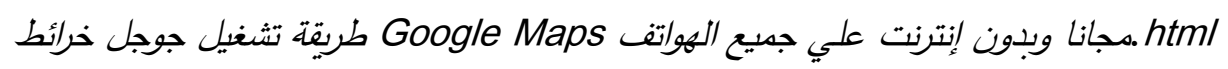

19- Why are maps important in day-to-day life? (2019) . www.quora.com, Retrieved 30-11.

20- Can Apple Maps ever be better than Google Maps?.(2019). www.quora.com, Retrieved 30-11. 21- www.wikiwand.com.

22- https:/support.google.com/maps/answer/144349?h/=ro\&ref topic=3092425.

23- http://maps.google.com/help/maps/education/. 
24- https://support.google.com/maps/answer/144349?h/=ar\&ref_topic=3092425\#zippy.

25- https://support.google.com/maps/answer/9625865?hl=ar\&ref_topic=3092425\&visit_id=63746296 4412191174-391898128\&rd=1.

26- https://support.google.com/maps/answer/6139433?h/=ar\&ref topic=3092425.

27-Planes For Google Earth Flight Simulator : Google Earth Blog. (2012) .available at : Gearthblog.com.

28-Smartphone (2019) : www.wikiwand.com, Retrieved 30-11.

\section{ملحق (1)}

قائمة المحكمين لقائمة المهارات

\begin{tabular}{|c|c|c|}
\hline الارجة العلمية & الأسم & r \\
\hline أستاذ مناهج وطرق تدريس الجغرافيا & أ.د/مجدي خير الدين كامل خير الدين & 1 \\
\hline أستاذ المناهج وطرق التدريس & أ.دد/حمدي محمد محمد البيطار & 2 \\
\hline أستاذ مناهج وطرق تدريس الجغرافيا المساعد & أ.م.د/جمال حسن السيد & 3 \\
\hline أستاذ تكنولوجيا التعليم المساعد & أ.م.د/ماريان ميلاد منصور & 4 \\
\hline
\end{tabular}

Received: April 2021

Accepted: June 2021 